\title{
The elusive gene for keratolytic winter erythema
}

\author{
P R Hull, MB BCh, MMed (Derm), FFDerm (SA), FRCPC, PhD (Med); A Hobbs, MSc; S Aron, MSc; M Ramsay, PhD \\ Peter Hull is from the Division of Dermatology, Department of Medicine, Royal University Hospital, University of Saskatchewan, Canada. Angela \\ Hobbs is from the Division of Human Genetics, School of Pathology, University of the Witwatersrand, Johannesburg, South Africa. Shaun Aron \\ is from Wits Bioinformatics, University of the Witwatersrand, South Africa. Michèle Ramsay is from the Division of Human Genetics, School of \\ Pathology and the Sydney Brenner Institute of Molecular Bioscience, University of the Witwatersrand, Johannesburg, South Africa.
}

Corresponding author: P R Hull (peter.hull@usask.ca)

Keratolytic winter erythema (KWE), also known as Oudtshoorn skin disease, is characterised by a cyclical disruption of normal epidermal keratinisation affecting primarily the palmoplantar skin with peeling of the palms and soles, which is worse in the winter. It is a rare monogenic, autosomal dominant condition of unknown cause. However, due to a founder effect, it occurs at a prevalence of $1 / 7200$ among South African Afrikaans-speakers. In the mid-1980s, samples were collected from affected families for a linkage study to pinpoint the location of the KWE gene. A genome-wide linkage analysis, using microsatellite markers, identified the KWE critical region on chromosome 8p23.1-p22. Subsequent genetic studies focused on screening candidate genes in this critical region; however, no pathogenic mutations that segregated exclusively with KWE were identified. The cathepsin B (CTSB) and farnesyl-diphosphate farnesyltransferase 1 (FDFT1) genes revealed no potentially pathogenic variants, nor did they show differential gene expression in affected skin. Mutation detection in additional candidate genes also failed to identify the KWE-associated variant, suggesting that the causal variant may be in an uncharacterised functional region. Bioinformatic analysis revealed highly conserved regions within the KWE critical region and a custom tiling array was designed to cover this region and to search for copy number variation. Although the study did not identify a variant that segregates exclusively with KWE, it provided valuable insight into the complex KWE-linked region. Next-generation sequencing approaches are being used to comb the region, but the causal variant for this interesting hyperkeratotic palmoplantar phenotype still remains elusive.

S Afr Med J 2013;103(12 Suppl 1):961-965. DOI:10.7196/SAMJ.7253 


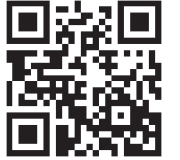

Dermatologists in South Africa (SA) have long been aware of a novel inherited skin disease, which was prevalent in families originating in the Oudtshoorn district of the Eastern Cape. In 1977, a meeting hosted by Professor Trefor Jenkins was held in Johannesburg. Based on the discussions and in collaboration with other dermatologists in SA, George Findlay authored the first publication detailing the clinical features and naming this novel genodermatosis, keratolytic winter erythema (KWE) or Oudtshoorn skin. ${ }^{[1]} \mathrm{He}$ later added the latinised variation, erythrokeratolysis hiemalis. ${ }^{[2]}$ Trefor Jenkins guided the extensive genealogical work by his PhD student, Peter Hull, who demonstrated that families with KWE residing in both the Oudtshoorn and Genadendal districts could be traced back to a single founder individual born in France. For several decades, scientists and students in the Division of Human Genetics, University of the Witwatersrand (Wits), and their collaborators have been searching for the mutation which is responsible for this fascinating skin disorder (Fig. 1). ${ }^{[1,3-9]}$ This is a brief overview of the search which also includes some novel data exploring the genomic architecture of the region where the causal mutation resides.

\section{Clinical features}

The most constant clinical sign of KWE is a cyclical, centrifugal peeling of the skin of the palms and soles. ${ }^{[1,3]}$ None of the other features of the disorder have been observed as isolated findings in the absence of this hand and foot involvement. In general, these changes are worse in the winter or with the changing of the seasons. In the mildest cases the only clinical evidence is scaling and redness of the web spaces of the fingers and toes. In some cases, there is a welldemarcated red boundary at the upper edge to the sole, or erythema may be observed which often involves the entire palm or sole. The initial clinical sign is the formation of superficial dry bullae that may be localised and well defined, or alternatively the skin appears opaque prior to it dissecting off to form a firm elastic peel. The peeling may occur at multiple sites, but rarely a single large 'bulla' may form a peel that involves the entire palm. The peeling proceeds centrifugally and is only arrested at major skin creases where a mild hyperkeratosis may persist for several weeks. The base revealed under the peeling skin is again red, while the papillary ridge pattern is retained (Fig. 2).
After a variable period of time, ranging from days to weeks, the cycle repeats itself. Hyperhidrosis is common during both the summer and winter and the sweat is often described as having a distinctive odour, which may be no more than the stench of macerated keratin. Although the palms and soles are most frequently affected, in some cases an annular erythema is noted involving the dorsum of the hands and forearms as well as the lower extremities including the buttocks.

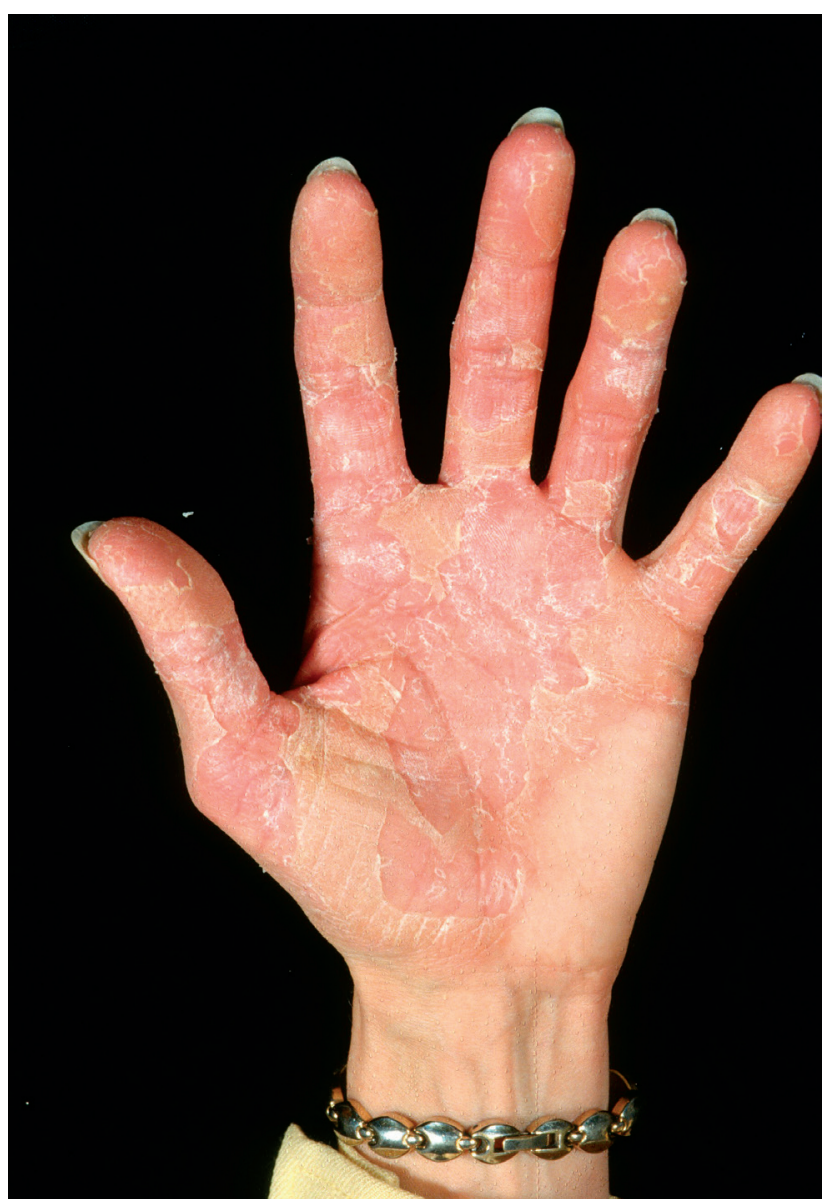

Fig. 2. Palm showing mild erythema and extensive peeling.

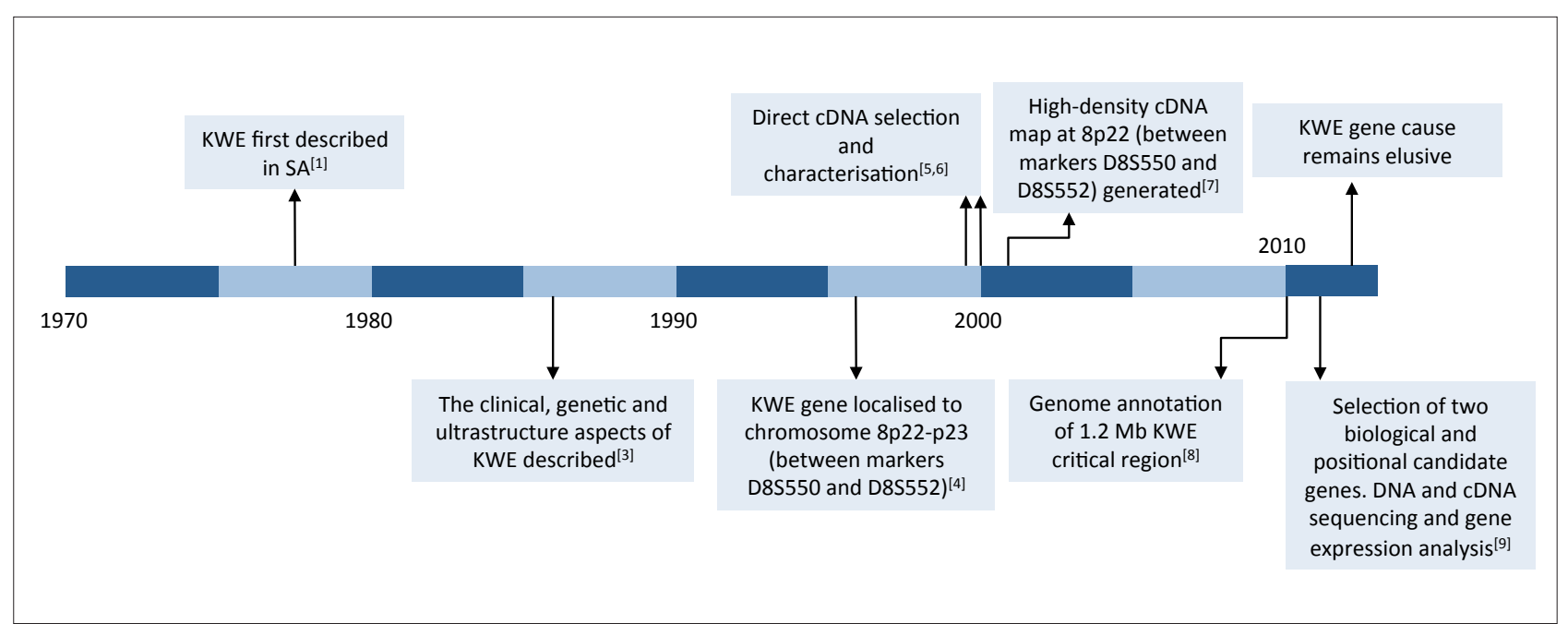

Fig. 1. Chronology of the research progress on searching for the keratolytic winter erythema (KWE)-causing mutation in South African families, showing the key contributions and publications over more than 4 decades. Although first formally described in 1977, the KWE causing founder mutation in South African families remains elusive. $c D N A=$ complementary DNA. ${ }^{[1,39]}$ 


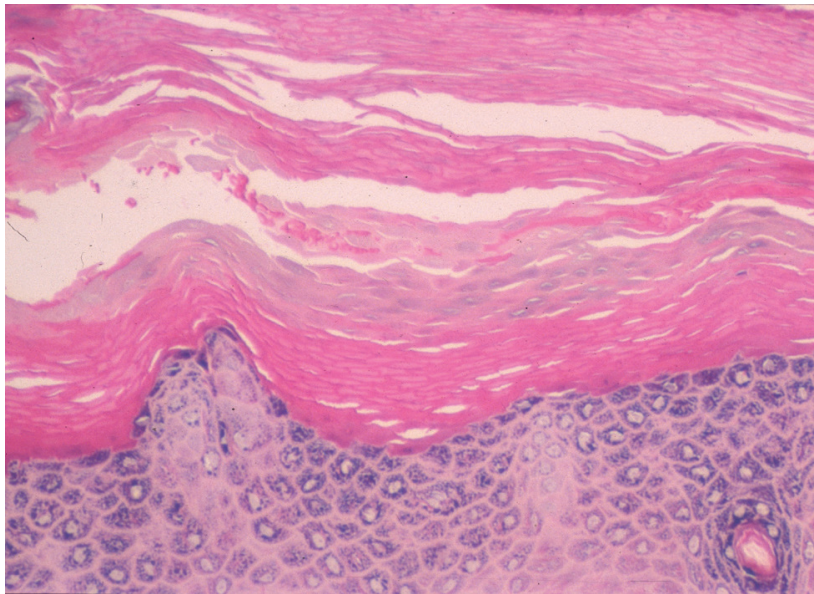

Fig. 3. Biopsy from the sole of the foot showing a recovered granular layer and a layer of parakeratosis sandwiched between the old orthokeratotic stratum corneum and a re-established orthokeratotic layer.

The trunk is only very rarely affected. These annular or rosette-like lesions have an outer expanding edge with a trailing circinate peel and central lamellar scale. They may expand over a period of $4-6$ weeks reaching up to $15 \mathrm{~mm}$ in size before healing. The condition improves with age and in adulthood only minimal scaling may be noted that is confined to the creases.

\section{Histopathology}

The changes are dynamic and are best illustrated by examining the annular lesions (Fig. 3). The earliest change appears be a focal area of mild spongiosis that spreads centrifugally. The affected keratinocytes fail to undergo normal keratinisation and are pushed upwards to form a compact parakeratotic layer beneath the previously normal orthokeratotic stratum corneum. There is rapid recovery of the epidermis and this is associated with a proliferation of basal keratinocytes and restoration of a granular layer and orthokeratotic horny layer. The parakeratotic scale then appears sandwiched between the original normal orthokeratotic layer and the newly regenerated orthokeratotic stratum corneum. The split occurs above the newly formed stratum corneum and forms a substantial peel. ${ }^{[1]}$

\section{Electron microscopy}

Only the annular rosette-like lesions have been examined. The spongiotic areas were extremely focal and found in the periphery of the erythema. They were characterised by greatly expanded intercellular spaces. The desmosomes occupied a central position in the interspace with taut, thin cytoplasmic strands connecting them to the opposing cells. Where the intercellular space was grossly expanded, the desmosomes appeared to be floating freely within the intercellular space without such connections. The desmosomes were morphologically normal and did not show internal separation. Apoptotic bodies rich in tonofilaments were not found. The internal structure of the keratinocytes showed striking changes in the mitochondria, including intracristal swelling with ballooning of the cristae and a denser matrix. Some of the keratinocytes showed larger electron lucid vacuoles that were arranged in a perinuclear position and these vacuoles appeared to develop from the progressive degeneration of the mitochondria. ${ }^{[3]}$

\section{Genetic studies}

KWE is an autosomal dominant, monogenic disorder with high penetrance. Families with KWE have been described mostly from SA where a genealogical study has identified a common ancestor and presumed founder, Captain Francois Renier Duminy, who was born in Lorient in France in $1747 .{ }^{[3]} \mathrm{He}$ was a mariner and visited the Cape of Good Hope several times, and later settled there with his wife, Johanna Margaretha (née Nöthling). A large German family with KWE has been described and two possible sporadic cases, one from Germany and the other from Denmark have been reported. ${ }^{[10,11]}$ Interestingly, there are no reported cases of KWE from France and it is possible that a different diagnosis may be attributed to the phenotype, which is likely to be quite rare.

The first sample collections for DNA studies in the KWE families started in the mid-1980s when several field trips were organised to the Oudtshoorn and Genadendal districts.

\section{Genetic linkage}

In the mid-1990s, a collaboration was initiated with Andre Reis' group from Germany to perform a genome-wide linkage study using microsatellite markers. Michelle Starfield, an MSc student in the Division of Human Genetics, the SA Institute for Medical Research (SAIMR) and Wits, embarked on research visits to the German laboratory which culminated in the identification of linkage to markers on chromosome 8p22-p23 in both the SA families and in the large German kindred. ${ }^{[4]}$ Haplotype analysis in the SA KWE families supported a common ancestry, while the German family demonstrated a different haplotype, suggesting a different origin. Based on these data and subsequent linkage analysis, attempting to narrow down the region of the KWE mutation, it was concluded that it is likely that through their single founder, all affected individuals in SA would have the same mutation.

\section{Candidate genes}

Once the KWE region had been mapped, it was important to identify candidate genes in the KWE critical region. Anton Bergheim, a PhD student at the SAIMR and Wits, and Silke Appel from Germany, used bacterial artificial chromosome (BAC) contigs of chromosome 8p22p23 and exome capture to select transcripts from this region. Twelve transcripts were identified by exon trapping, complementary DNA (cDNA) selection and sequence analyses, but no pathogenic mutations were identified in any of these transcripts. ${ }^{[7]}$ Further analysis of 16 candidate cDNA clones derived from this experiment was performed by Hugh Napier, an MSc student (unpublished data) but yielded no candidate mutations. In an attempt to narrow down the KWE region and pinpoint the causative gene more precisely, families with informative crossover events were investigated. As a result, the KWE critical area was narrowed to chromosome 8p23.1-p22 between, and including markers D8S1759 and D8S552, a region roughly1.26 Mb in size. The KWE critical region is flanked by two olfactory receptor/defensin repeat regions which are prone to non-homologous recombination resulting in large chromosomal rearrangements, including a common 4.7 $\mathrm{Mb}$ polymorphic 8p23 inversion. ${ }^{[12]}$

\section{Candidate gene analysis and gene expression}

The two genes identified within the KWE critical region as being the most likely candidates, based not only on chromosomal location but also on biological function, were the cathepsin $\mathrm{B}(C T S B)$ gene and the farnesyl-diphosphate farnesyltransferase 1 (FDFT1) gene. Mutation 
screening of the coding regions, 5' untranslated regions (UTRs) and intron/exon boundaries of both genes in genomic DNA and cDNA of patients affected with KWE identified no causal variants. To determine whether these genes had differential expression in KWEaffected individuals compared with unaffected controls, epidermal skin biopsies were taken from either the individuals' palms or soles. RNA was extracted from the skin samples and reverse-transcribed into cDNA, which was used as the template in quantitative real-time polymerase chain reaction analysis. The comparative $\Delta \Delta \mathrm{Ct}$ method was used to determine the target genes' relative expression in affected individuals. A marked increase in the expression of FDFT1 messenger RNA (mRNA) was observed in affected individuals. However, this increase was not statistically significant in the small sample size tested, although increased expression of the gene appeared to be correlated with the severity of the clinical presentation. An examination of the FDFT1 promoter failed to identify a KWE-restricted variant. ${ }^{[9]}$

\section{Novel data exploring highly conserved coding and non- coding regions and copy number variants}

As the examination of candidate genes in the critical region failed to identify KWE-associated variants, the possibility of the causal variant occurring in an uncharacterised functional region was investigated. Although variants within protein-coding genes are more likely to be implicated as disease-causing mutations, highly conserved, nonprotein-coding regions have been shown to be involved in gene regulation. Variations within these regions may directly affect the expression levels of nearby genes and be involved in the cause of diseases ${ }^{[13]}$ In addition to the presence of genetic variation in the form of microscopically visible chromosomal rearrangements and single nucleotide changes, there is an abundance of copy number variations (CNVs) of DNA segments throughout the genome. CNVs have been implicated as the cause of a number of genetic disorders ${ }^{[1,15]}$ and many CNV regions have been observed on chromosome 8p23.1 within the region of the $\beta$-defensin gene cluster. ${ }^{[16,17]} \mathrm{A}$ two-fold approach was aimed at examining the critical region for both highly conserved coding and non-coding sequences as well as CNVs and to characterise these regions further to determine if they may be functional and play a role in the molecular aetiology of KWE. This work was the research of an MSc student, Shaun Aron. ${ }^{[8]}$

\section{Bioinformatic analysis of novel regions of interest}

To identify previously uncharacterised functional sequences within the KWE critical region, a comparative genomics approach was used to identify regions that exhibited high levels of conservation across a range of evolutionarily diverse organisms. A multiple sequence alignment of the critical region on chromosome 8:11 317148 - 12899 353 (HG18) for eight evolutionarily diverse organisms was generated. Regions $\geq 100 \mathrm{bp}$ that showed a conservation score of $>70 \%$ were identified and further characterised for functional signatures via in silico prediction methods for gene structures, transcription factor binding sites and RNA secondary structures.

Eight distinct regions of high conservation were identified. Six were found within non-coding regions of the genome, while the remaining two were within a previously annotated open reading frame. Each of these regions ranged in size from $100 \mathrm{bp}$ to $406 \mathrm{bp}$ and showed high levels of conservation in at least six of the species. Of the eight regions assessed for potential functional roles, only one presented as a possible functional candidate for KWE. The region was highly conserved across all eight species and presented as a possible single-exon gene structure. Further examination revealed that there was an exact match of the region to an mRNA sequence previously described as Homo sapiens deubiquitinating enzyme 3 (DUB3). At the time of the study, DUB3 was not mapped to chromosome 8; however, it was known to belong to a group of deubiquitinating enzymes responsible for the cleavage of ubiquitin-conjugated proteins.

Deubiquitinating enzymes function by removing the covalently attached ubiquitin from proteins, thereby controlling substrate activity and/or abundance. DUB3 is a cytokine inducible protein expressed transiently and is thought to play a role in blocking cell proliferation and inducing apoptosis through the ubiquitin pathway. The molecular functioning of DUB3 therefore presented the gene as a likely novel candidate gene for the KWE phenotype. Subsequently, during the period of the study, DUB3 was annotated to this conserved region as a single exon gene lacking a 5' or $3^{\prime}$ UTR and encoding a protein of 530 amino acids. This annotation correlated perfectly with the gene prediction obtained in the study. The gene was sequenced in a subset of KWE-affected individuals to identify a possible mutation within the coding region; however, all six of the variants identified were observed at a frequency of $>70 \%$ in a corresponding control group and therefore unlikely to be causal.

\section{Copy number variation}

A custom tiling array (Roche NimbleGen) containing roughly 385000 probes at a resolution of $11 \mathrm{bp}$ was designed based on an extended region encompassing the inverted region on chromosome $8 \mathrm{p}$ and the KWE-linked critical region between markers D8S1759 and D8S552 (chromosome 8: 11415050 - 12886829 (HG18)). Samples were collected from 10 individuals from four families of which five were KWE-affected and five were unaffected.

The comparative genome array data were normalised using the Qspline method and two datasets were generated for further analysis. The first was the complete normalised dataset consisting of 384702 data points and the second was a transformed window-averaged dataset generated by averaging the intensities across $100 \mathrm{bp}$ windows to analyse larger regions of CNV. All known protein-coding genes in the region were examined for CNVs in both the KWE-affected and unaffected samples. To compare the CNVs between the affected and unaffected individuals, a pairwise comparison was conducted between the affected individual and their unaffected relative. CNVs that were present in both the affected and unaffected individuals were excluded since they are unlikely to be involved in KWE. All regions that exhibited CNV only in the affected individuals were further assessed to determine if they might play a role in the molecular aetiology of KWE.

None of the previously studied candidate genes showed CNV. The regions that exhibited CNVs either occurred within known low-copy repeat regions, i.e. $\beta$-defensin and olfactory receptor gene clusters or within genes outside of the KWE critical region. Although higher copy numbers of the repeat unit encompassing the $\beta$-defensin cluster has been implicated in inflammatory skin disorders such as psoriasis, no consistent $\mathrm{CNV}$ at this region was observed across all five KWE affected individuals. The genes found outside of the KWE region that showed CNV included MSRA, RP1L1, PINX1 and XKR6. These genes were further investigated, but their molecular function failed to suggest any link to the pathogenesis of KWE.

A number of smaller CNVs were identified within non-coding regions. Two of the most plausible CNVs, possibly involved in a position effect were those identified upstream of the two strong KWE candidate genes, CTSB and DUB3 (USP17L2). The CNV upstream of CTSB is located $8873 \mathrm{bp}$ upstream of the gene and was found to be duplicated in $3 / 5$ affected individuals with no variation observed in 
the control samples. It is possible that this region may harbour some regulatory elements involved in the control of expression of either CTSB or another gene in the region. Similarly, the CNV of $24 \mathrm{bp}$ found $38007 \mathrm{bp}$ upstream of DUB3, which was deleted in $3 / 5$ affected individuals could possibly be involved in the regulation of DUB3. Most disease causing CNVs involving regulatory regions encompass large regions of regulatory sequences ranging in size from $\mathrm{kb}$ to $\mathrm{Mb}$. It is therefore unlikely that these regions alone would be involved in the molecular aetiology of KWE, but they do present as interesting areas for further investigation.

Although the study did not identify the causal variant for KWE, it served as an exploratory method to provide valuable insight into the complex KWE-linked region and provide potential regions of interest for further study.

\section{Treatment}

The results of treatment are disappointing and highly variable. Topical steroids seem to be helpful in some cases, but in others appear to aggravate the condition. In one patient, systemic steroids temporally resolved the annular lesions. Most promising has been the work by Amin et al. ${ }^{[18]}$ reporting on the use of photodynamic therapy (PDT) for the treatment of systematic KWE. Methyl 5- aminolevulinate was used as the photosensitiser and only two treatments were given, one week apart. The patch was exposed to $37 \mathrm{~J} / \mathrm{cm}^{2}$ with $630 \mathrm{~nm}$ red light, 3 hours after the application of the photosensitiser. The treated area remained disease-free at 24 months. Certain features of KWE lesions, such as a defect in the stratum corneum and an increased rate of cell proliferation, may explain the success of PDT as a possible treatment for the disease. These features could lead to the selective uptake of the photosensitiser into abnormal cells. PDT may be a successful treatment for KWE, but is yet to be widely tested. ${ }^{[18]}$

\section{Next steps}

The mutation responsible for KWE remains elusive. The phenotype presents periodically, with some clinical differences at different time points of a life and across individuals, its effects appear to be modulated by environmental and physiological factors such as cooler temperature, systemic infection and pregnancy, and the cellular phenotype is propagated from cell to cell, possibly via gap junctions. Many avenues to the identification of the KWE mutation have been explored including candidate gene mutation detection, gene expression changes, the identification of conserved regions in the KWE critical region and CNV regions; yet none has pinpointed a mutation restricted to affected individuals. New technologies are presenting novel high-throughput biological approaches including target genome capture and next-generation sequencing and extensive quantitative transcriptome analysis. Challenges remain in identifying which genetic variants are potentially causal and in isolating relevant cells at appropriate stages of pathology for detailed transcriptome research. Although there is still no effective treatment, photodynamic therapy has a disease-modifying effect, but needs to be better understood. KWE remains a genetic and molecular riddle to be solved.

Acknowledgement. Research Support was provided by the Canadian Dermatology Foundation, SA National Research Foundation, SA National Health Laboratory Service Research Trust, University of the Witwatersrand as well as The Canadian Foundation of Dermatology.

1. Findlay GH, Nurse GT, et al. Keratolytic winter erythema or 'Oudtshoorn skin': A newly recognised inherited dermatosis prevalent in South Africa. S Afr Med J 1977;52(22):871-874.

2. Findlay GH, Morrison JG. Erythrokeratolysis hiemalis - keratolytic winter erythema or 'Oudtshoorn Skin'. A new epidermal genodermatosis with its histological features. Br J Dermatol 1978;98(5):491-495.

Hull P. Keratolytic Winter Erythema (Oudtshoorn Disease): Clinical, Genetic and Ultraspectural Hull P. Keratolytic Winter Erythema (Oudtshoorn Disease): Clinical,
Aspects. PhD thesis. Johannesburg: University of the Witwatersrand, 1986.

4. Starfield M, Hennies $\mathrm{HC}$, et al. Localization of the gene causing keratolytic winter erythema to chromosome 8p22-p23, and evidence for a founder effect in South African Afrikaans-speakers. Am J Hum Genet 1997;61(2):370-378. [http://dx.doi.org/10.1086/514848]

5. Napier HRL. The Characterisation of Candidate cDNA Clones for the Gene Causing Keratolytic Winter Erythema. BSc (Hons) Research report. Johannesburg: University of the Witwatersrand, 1999.

Ogilvie EM. Characterisation of Candidate Keratolytic Winter Erythema Clones from a Direct Selected Fetal Brain cDNA Library. MSc dissertation. Johannesburg: University of the Witwatersrand, 2000.

7. Appel S, Filter M, Reis A, et al. Physical and transcriptional map of the critical region for keratolytic winter erythema (KWE) on chromosome 8p22-p23 between D8S550 and D8S1759. Eur J Hum Genet 2002;10(1):17-25. [http://dx.doi.org/10.1038/s.ejhg.5200750]

8. Aron S. Genome Annotation of the $1.2 \mathrm{Mb}$ Region on Chromosome 8p22-23.1 Harbouring the Gene for Keratolytic Winter Erythema (KWE).MSc disertation John

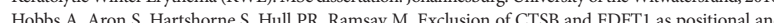
Hobbs A, Aron S, Hartshorne S, Hull PR, Ramsay M. Exclusion of CTSB and FDFT1 as positional an functional candidate genes for keratolytic winter erythema (KWE). J Dermatol Sci 2012;65(1):58-62.
[http://dx.doi.org/10.1016/j.jdermsci.2011.08.008] [http://dx.doi.org/10.1016/j.jdermsci.2011.08.008]

0. Danielsen AG, Weismann K, Thomsen HK. Erythrokeratolysis hiemalis (keratolytic winter erythema) A case report from Denmark. J Eur Acad Dermatol Venereol 2001;15(3):255-256. [http://dx.doi org/10.1046/j.1468-3083.2001.00258.x]

11. Krahl D, Sigwart A, Hartschuh W, Anton-Lamprecht I, Petzoldt D. Erythrokeratolysis hiemalis. Erythematosquamous genetic dermatosis with seasonal manifestation. Hautarzt 1994;45(11):776-779.

12. Giglio $\mathrm{S}$, Calvari V, Gregato $\mathrm{G}$, et al. Heterozygous submicroscopic inversions involving olfactory receptorgene clusters mediate the recurrent $\mathrm{t}(4 ; 8)(\mathrm{p} 16 ; \mathrm{p} 23)$ translocation. Am J Hum Genet 2002;71(2):276-285 [http://dx.doi.org/10.1086/341610]

3. Stranger BE, Nica AC, Forrest MS, et al. Population genomics of human gene expression. Nat Genet 2007;39(10):1217-1224. [http://dx.doi.org/10.1038/ng2142]

14. Balikova I, Martens K, Melotte C, et al. Autosomal-dominant microtia linked to five tandem copies of a copy-number-variable region at chromosome 4p16. Am J Hum Genet 2008;82(1):181-187. [http://dx.doi. org/10.1016/j.ajhg.2007.08.001]

15. Thienpont $B$, de Ravel T, van Esch $H$, et al. Partial duplications of the ATRX gene cause the ATR-X . Thienpont B, de Ravel T, van Esch H, et al. Partial duplications of the ATRX gene cause the
syndrome. Eur J Hum Genet 2007;15(10):1094-1097. [http://dx.doi.org/10.1038/sj.ejhg.5201878]

16. Barber JC, Maloney VK, Huang S, et al. 8p23.1 duplication syndrome; a novel genomic condition with unexpected complexity revealed by array CGH. Eur J Hum Genet 2008;16(1):18-27. [http://dx.doi.
und org/10.1038/s.ejhg.5201932]

17. Hollox EJ, Armour JA, Barber JC. Extensive normal copy number variation of a $\beta$-defensin antimicrobialgene cluster. Am J Hum Genet 2003;73(3):591-600. [http://dx.doi.org/10.1086/378157]

18. Amin AN, DeGiovanni CV, Farrant PB, Hull PR, Woollons A. Photodynamic therapy for the treatment of keratolytic winter erythema. Clin Exp Dermatol 2011;36(6):668-669. [http://dx.doi.org/10.1111/j.13652230.2010.03927.x]

Accepted 22 August 2013. 